SEP 111992

\title{
STEAM GASIFICATION OF CARBON: CATALYST PROPERTIES
}

Reporting period: March 15, 1992 - June 14, 1992

Prepared for U.S. D.O.E. Grant Number: DE-FG22-88PC 88915

University of Colorado Proposal Number: 0188.11.0115B

Project officer: Kamalendu Das

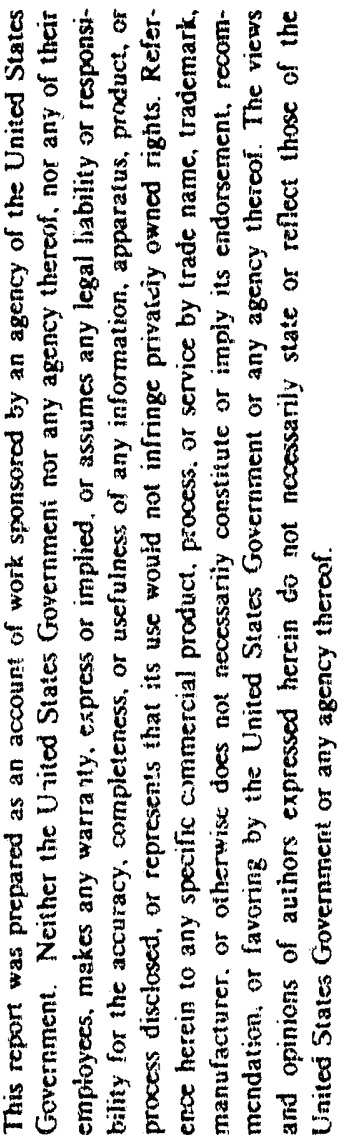

\section{Principal Investigator:}

Professor John L. Falconer

July 6, 1992 


\section{Table of Contents}

\section{Page}

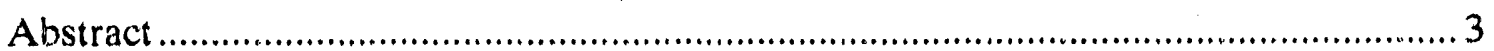

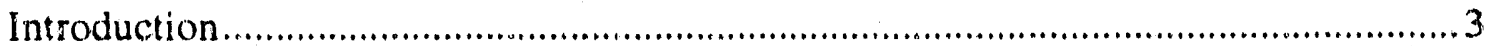

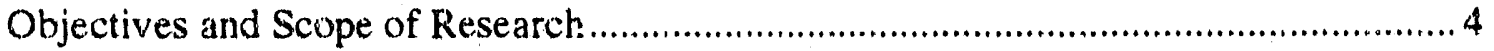

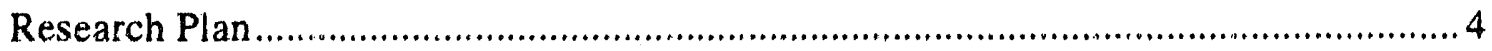

Project Status, Accomplishments, and Technical Discussion...................................6 


\begin{abstract}
This research uses several techniques to measure the concentration of catalyst sites and determine their stoichiometry for the catalyzed gasification of carbon. Both alkali and alkaline earth oxides are effective catalysts for accelerating the gasification rate of coal chars, but only a fraction of the catalyst appears to be in a form that is effective for gasification, and the composition of that catalyst is not established. Transient techniques, with ${ }^{13} \mathrm{C}$ labeling, are being used to study the surface processes, to measure the concentration of active sites, and to determine the specific reaction rates.

We have used secondary ion mass spectrometry (SIMS) for both high surface area samples of carbon/alkali carbonate mixtures and for model carbon surfaces with deposited alkali atoms. SIMS provides a direct measure of surface composition. The combination of these results can provide knowledge of catalyst dispersion and composition, and thus indicate the way to optimally utilize carbon gasification catalysts.
\end{abstract}

\title{
INTRODUCTION
}

Coal gasification by steam is of critical importance in converting coal to gaseous products $\left(\mathrm{CO}, \mathrm{H}_{2}, \mathrm{CO}_{2}, \mathrm{CH}_{4}\right)$ that can then be further converted to synthetic natural gas and higher hydrocarbon fuels. Alkali and alkaline earth metals (present as oxides) catalyze coal gasification reactions and cause them to occur at significantly lower temperatures. A more fundamental understanding of the mechanism of the steam gasification reaction and catalyst utilization may well lead to better production techniques, increased gasification rates, greater yields, and less waste.

We are studying the gasification of carbon by steam in the presence of alkali and alkaline earth oxides, using carbonates as the starting materials. Carbon dioxide gasification $\left(\mathrm{CO}_{2}+\mathrm{C}->\right.$ $2 \mathrm{CO}$ ) has been studied in some detail recently, but much less has been done on the actual steam gasification reaction, which is the main thrust of cur work. In particular, the form of the active catalyst compound during reaction is still questioned and the dependence of the concentration of active sites on reaction parameters is not known. Until recently, no measurements of active site concentrations during reaction had been made. We have recently used transient isotope tracing to determine active site concentration during $\mathrm{CO}_{2}$ gasification.

We are investigating the mechanism and the concentration of active sites for steam gasification with transient isotopic tracing. For this technique, the reactant feed is switched from $\mathrm{H}_{2} \mathrm{O}$ to isotopically-labeled water at the same concentration and flow rate. We can then directly measure, at reation conditions, the concentration of active catalytic sites, their kinetic rate constants, and the presence of more than one rate constant. This procedure allows us to obtain transient kinetic data without perturbing the steady-state surface reaction. A mass spectrometer with computer interface allows each of the approprate mass peaks to be followed in time. The changes in catalytic site concentrations and their rate constants will be measured as a function of reaction time, initial catalyst concentration, and reaction temperature. Potassium and calcium salts are being used.

The composition of the active catalyst is being studied with secondary ion mass spectrometry (SIMS) for catalyst-carbon mixtures, after high temperature treatment in steam. Model catalyst-carbon surfaces are being prepared so that a planar surface can be obtained for SIMS analysis. Details of the local bonding in reacting solids can also be obtained with SIMS. 
Scanning tunneling microscopy (STM), a new technique that can obtain atomic images of surfaces with excellent resolution, is being used to determine the location and distribution of catalysts on carbon surfaces. This will be a direct measure of catalyst location and size. By combining SIMS, STM, and transient isotope tracing, we will obtain a detailed understanding of the utilization of the catalyst, its location on the surface, and its chemical form. This information will help in improving catalyst utilization in steam gasification of coal.

\section{OBJECTYYES AND SCOPE OF RESEARCH}

The objectives of this research are to determine the catalyst composition and concentration for metal oxide (potassium and calcium oxides) catalyzed gasification of carbon. The scope of this research involves determining:

- The concentration of active catalytic sites (and thus the dispersion), at reaction conditions, as a function of temperature, carbon burnoff, catalyst loading, and catalyst type.

- The location of catalyst, on the surface, on an atomic scale.

- The composition of the catalyst.

- The site-specific rate constant for steam gasification.

- Insight into the reaction mechanism.

- How to maximize catalyst dispersion.

Knowledge of how catalyst dispersion depends on reaction conditions will provide a basis for making optimal use of the catalyst in steam gasification of coal.

\section{RESEARCH PLAN}

The catalyst composition, concentration, and location for catalyzed gasification of carbon and coal char by steam will be investigated for both alkali metal and a!kaline earth carbonates. Isotope tracing will measure the concentration of active catalyst sites, their specific rate constant, and the dependence of sites on initial catalyst loading, carbon conversion (burnoff), and temperature. Transient isotope tracing, secondary ion mass spectrometry (SIMS), and scanning tunneling microscopy (STM), will be used to obtain a complete picture of the catalysts at reaction conditions.

Carbon black, coal chars, and graphite will be used in the transient tracing experiments. In the SIMS and STM experiments, graphite and carbon deposited on a metal surface will be models of coal char. These forms will allow better utilization of these sensitive surface techniques.

Isotope Tracing Experiments: Transient isotope tracing allows the rate constant and the effective active site concentration to be uncoupled so that a direct measure of reactive site concentration can be obtained. The presence of more than one type of site can also be determined. Isotorse tracing can also identify the rate-determining step. We are applying isotope tracing to catalyzed gasification of carbon by steam. A small amount of carbon, impregnated with $\mathrm{K}_{2} \mathrm{CO}_{3}$, will be heated to reaction conditions in a $\mathrm{H}_{2} \mathrm{O} / \mathrm{He}$ steam. The $\mathrm{H}_{2}, \mathrm{CO}$, and $\mathrm{CO}_{2}$ reaction products and unreacted $\mathrm{H}_{2} \mathrm{O}$ will be detected with a quadrupole mass spectrometer located immediately downstream from the reactor. Transient mass signals will be measured as $\mathrm{H}_{2}^{18} \mathrm{O}$ replaces $\mathrm{H}_{2}^{16} \mathrm{O}$ in the feed stream (step increase in $\mathrm{H}_{2}^{18} \mathrm{O}$ and step decrease in $\mathrm{H}_{2}^{16} \mathrm{O}$. A computer interfaced to the mass spectrometer allows $\mathrm{C}^{18} \mathrm{O}, \mathrm{C}^{16} \mathrm{O}, \mathrm{C}^{16} \mathrm{O}_{2}, \mathrm{C}^{18} \mathrm{O}_{2}, \mathrm{C}^{16} \mathrm{O}^{18} \mathrm{O}, \mathrm{H}_{2}^{16} \mathrm{O}, \mathrm{H}_{2}^{18} \mathrm{O}$ and $\mathrm{H}_{2}$ products to be detected simultaneously as a function of time. As soon as the ${ }^{18} \mathrm{O}$ products reach steady state, the 
$\mathrm{H}_{2}^{18} \mathrm{O}$ will be replaced by $\mathrm{H}_{2}^{16} \mathrm{O}$ to repeat the process.

Since the partial pressure of steam is unchanged when the isotopes are switched, steadystate reaction is not disturbed, but the isotopic composition of the product mix must adapt itself to the isotopic composition of the feed. A kinetic isotope effect may change the steady-state reaction site, however. This relaxation provides mechanistic information.

These transient isotope experiments will be carried out for a range of reaction conditions to determine what fraction of the catalyst is active for reaction:

- Initial catalyst weight loading will be varied to determine how active site concentration varies with catalyst concentration. The catalyst/carbon ratio will vary with carbon conversion, and thus the concentration of active sites will also be measured as a function of burnoff.

- Reaction temperature will be varied to determine how the number of active sites varies with temperature. At higher temperatures, the loss of $\mathrm{K}$ metal can be checked by remeasuring active site concentrations at lower temperatures.

- Both $\mathrm{K}$ and Ca catalysts will be used. A better understanding of the role of the catalyst can be obtained by determining the differences between alkali and alkaline earth catalysts.

Secondary Ion Mass Spectrometry (SIMS): Secondary ion mass spectrometry consists of bombarding a surface under UHV conditions with energetic primary ions (typically $\mathrm{Ar}^{+}$) at sufficient atomic motion to eject particles from the surface. Some of the ejected particles are ionic (secondary ions) and are mass analyzed by a mass spectrometer to give the secondary ion mass spectrum. To prevent significam impact damage to the surface, the experiment is normally conducted in the static mode using a low primary ion current density (typically $1-10 \times 10^{-9} \mathrm{~A} / \mathrm{cm}^{2}$ ).

SIMS will be used to probe the identity and reaction chemistry of the species present during catalytic steam gasification. SIMS has unique potential for this since molecular species can be ejected from the surface with their original structure preserved and can therefore be identified directly from the SIMS spectrum.

SIMS will also be used to probe the local chemical environment of surface components. Ejected molecular cluster ions form by reassociation of independently-ejected surface components that are within close proximity of each other (lypically 1-5 A). These molecular clusters are thus indicative of a close proximity between the cluster components on the original surface. This information is essential for a complete understanding of the surface reaction chemistry. SIMS can selectively probe the local environment of such components through the formation of cluster ions containing these components. These capabilities of SIMS for probing the identity, reaction chemistry, and local chemical environment of surface species will be exploited to develop a better understanding of catalytic gasification chemistry.

Carbonate-carbon samples will be examined by SIMS before and after exposure to reaction conditions to identify the chemical species present on the active surface and their local chemical environment. The effects of reaction temperature, burnoff time, and catalyst loading will be studied for both potassium and calcium catalysts.

Model carbon surfaces will be prepared to produce a cleaner and more well defined carbon surface than that of the carbon black samples, which contain hydrocarbon and oxygenated impurities. Since our previous work shows that the metal-carbonate catalysts thermally decompose to oxides, we will attempt to place the active catalyst on the carbon surface by first vapor depositing, in UHV, the alkali metal and then forming the alkali metal oxide by exposure to oxy- 
gen. The nature of the interaction of water with both calcium and potassium oxides on such model surfaces will be determined by SIMS analysis before and during exposure to a $\mathrm{H}_{2} \mathrm{O}$ beam in UHV. The effects of temperature, metal oxide coverage, $\mathrm{H}_{2} \mathrm{O}$ pressure, and exposure time to the $\mathrm{H}_{2} \mathrm{O}$ beam will be studied.

Scanning Tunneling Microscopy (STM): Since the dispersion of the catalyst can significantly affect the reaction rate, we will measure catalyst particle size to determine how dispersion depends on catalysi concentration, surface structure, and temperature. We will also determine the atomic location of the catalyst on the surface. By using graphite with few grain boundaries and thin layers of carbon deposited onto a single crystal metal, we will study the influence of surface structure.

Scanning tunneling microscopy (STM) is nondestructive and yields real-space imaging on an atomic dimension. Experimentally, electric current flows between an atomically sharp needle and the surface of interest. The extreme sensitivity of the tunneling current to tunneling distance is the basis of operation. Tunneling current decreases by roughly ten times for each increase of $0.1 \mathrm{~nm}$ spacing. If the needle is scanned laterally across the surface of interest, while the distance between needie and surface is adjusted to maintain a constant tunnel current, the topography of the surface can be obtained. Lateral resolution of $0.1 \mathrm{~nm}$ and height corrugations of $0.01 \mathrm{~nm}$ have been obtained. The unique advantages of STM are its atomic resolution (individual atoms can be detected) and its direct measure of surface atom location and structure.

\section{PROJECT STATUS, ACCOMPLISHMENTS, AND TECHNICAL DISCUSSION}

All our efforts were devoted to putting the STM system into operation for obtaining images of carbon surfaces onto which catalysts have been deposited. Significant gains were made in solving a number of electronic problems and suitable repairs were made. However, stable tunneling currents were not obtained after two months of efforts. After numerous consultation with the microscope and the electronics manufacturers, the electronics were returned to the manufacturer for evaluation. 

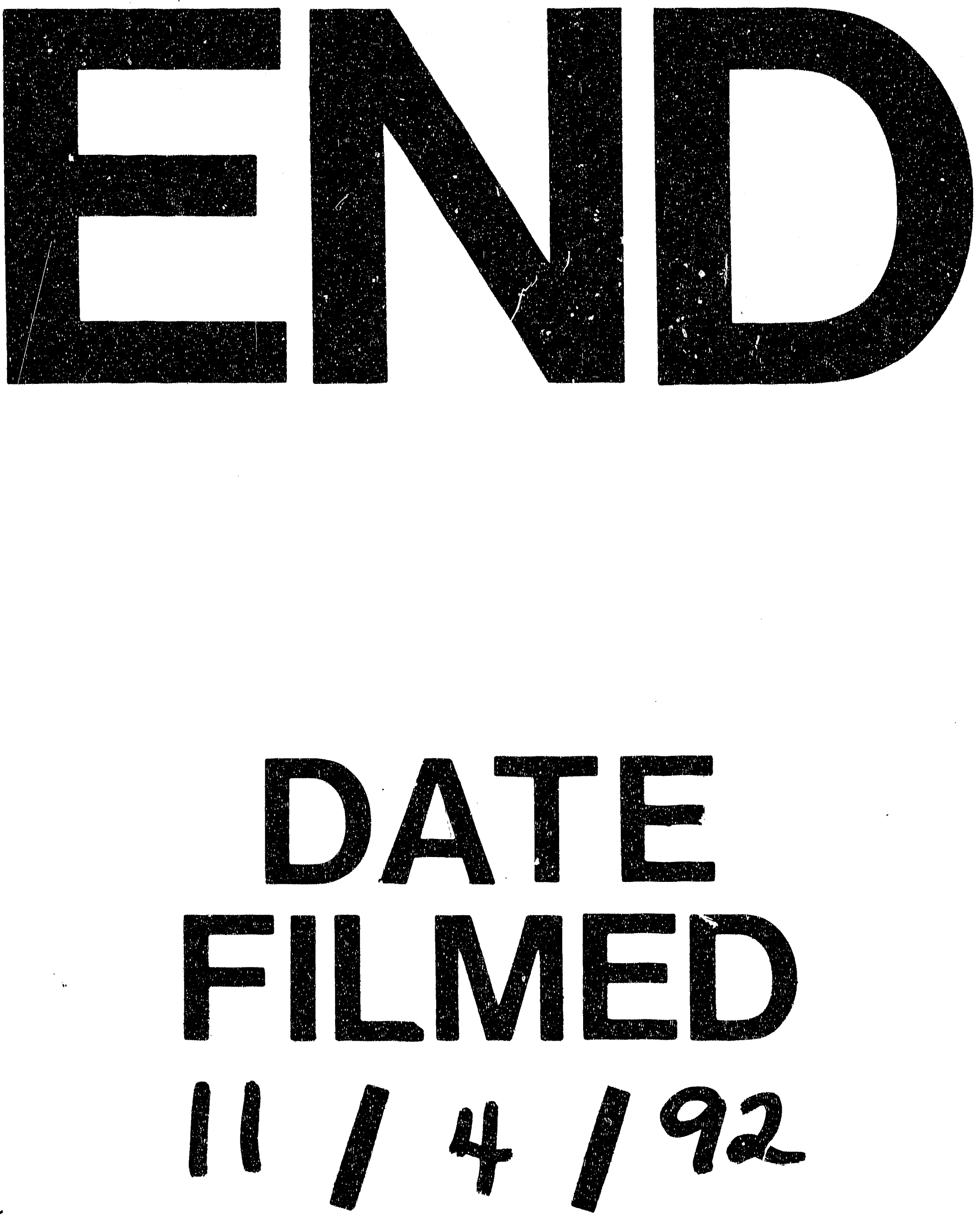

4
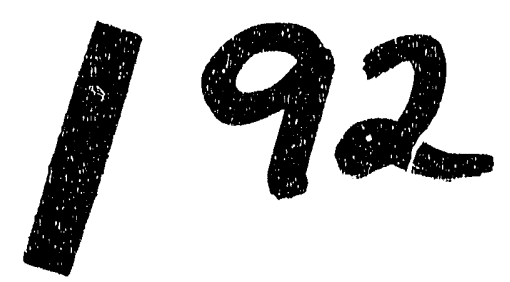

1 
